\title{
Synthesis and stacked conformations of symmetrical and unsymmetrical oligo-ureas of metaphenylenediamine
}

\author{
Jonathan Clayden, * Loïc Lemiègre and Madeleine Helliwell \\ School of Chemistry, University of Manchester, Oxford Road, Manchester M13 9PL, UK
}

\section{SUPPLEMENTARY INFORMATION}

Pages S1-S11 Experimental data for the preparation of 9b-d, 10b-c, 11b-e, 16 and 17b-g

Pages S12-S13 ${ }^{1} \mathrm{H}$ and ${ }^{13} \mathrm{C}$ NMR spectra of 11c

Pages S14-S $15{ }^{1} \mathrm{H}$ and ${ }^{13} \mathrm{C}$ NMR spectra of $\mathbf{1 6}$

General experimental details have been reported before. ${ }^{1}$ Flash chromatography refers to chromatography carried out using the method of Still, Kahn and Mitra. ${ }^{2}$

1. Clayden, J.; Vallverdú, L.; Helliwell, M., Org. Biomol. Chem. 2006, 4, 2106.

2. Still, W. C.; Kahn, M.; Mitra, A., J. Org. Chem. 1978, 43, 2923. 
$N, N$, $N$ ', $N$ ','Tetramethyl-1-(2,6-diethylphenylureyl)-3-(2-bromophenylureyl)benzene 9b. -By the

method used for 9a, 3-nitrophenylisocyanate (837 mg, $5.10 \mathrm{mmol}, 1.2 \mathrm{eq})$ and 2,6-diethylaniline (700 $\mu \mathrm{L}, \quad 4.25 \mathrm{mmol}, 1 \mathrm{eq})$ gave, after the hydrogenation, $1.02 \mathrm{~g}$ of crude material. 2Bromophenylisocyanate (890 $\mu \mathrm{L}, 7.20 \mathrm{mmol}, 2.0$ eq.) was used instead of phenylisocyanate. The methylation was carried out in THF (30 mL) with NaH 60\% in mineral oil (720 mg, $18.0 \mathrm{mmol}, 5$ eq.) and methyl iodide (1.12 mL, $18.0 \mathrm{mmol}, 5$ eq.). After same treatment purification by flash chromatography on silica $\left(90 / 10: \mathrm{CH}_{2} \mathrm{Cl}_{2} / \mathrm{MeOH}\right)$ and recrystallisation in $\mathrm{Et}_{2} \mathrm{O} /$ petroleum ether gave urea 9b $(1.51 \mathrm{~g}, 66 \%)$ as a white powder. m.p. : $134^{\circ} \mathrm{C}$. IR $v_{\max } \mathrm{cm}^{-1}: 2965,2934,1656,1597,1428$, 1351. ${ }^{1} \mathrm{H}$ NMR $\left(500 \mathrm{MHz}, \mathrm{CDCl}_{3}\right) \delta(\mathrm{ppm}): 1.05(6 \mathrm{H}, \mathrm{t}, J=7.5 \mathrm{~Hz}), 2.10(2 \mathrm{H}, \mathrm{m}), 2.28(2 \mathrm{H}, \mathrm{m}), 2.80$ $(3 \mathrm{H}, \mathrm{s}), 2.94(3 \mathrm{H}, \mathrm{s}), 3.02(3 \mathrm{H}, \mathrm{s}), 3.07(3 \mathrm{H}, \mathrm{s}), 5.84(1 \mathrm{H}, \mathrm{s}), 6.31(1 \mathrm{H}, \mathrm{d}, J=7.7 \mathrm{~Hz}), 6.52(1 \mathrm{H}, \mathrm{d}, J=$ $7.8 \mathrm{~Hz}), 6.61(1 \mathrm{H}, \mathrm{d}, J=7.8 \mathrm{~Hz}), 6.75(4 \mathrm{H}, \mathrm{m}), 6.87(1 \mathrm{H}, \mathrm{t}, J=7.5 \mathrm{~Hz}), 6.95(1 \mathrm{H}, \mathrm{t}, J=7.5 \mathrm{~Hz}), 7.28$ $(1 \mathrm{H}, \mathrm{d}, J=7.9 \mathrm{~Hz}) .{ }^{13} \mathrm{C} \mathrm{NMR}\left(75 \mathrm{MHz}, \mathrm{CDCl}_{3}\right) \delta(\mathrm{ppm}): 14.1,23.3,38.3,38.5,39.6,40.8,122.5$, $123.1,123.2,123.4,125.7,127.3,127.4,127.6,128.8,129.8,133.1,140.6,141.2,143.6,145.4,145.6$, 160.1, 161.5. MS (ESI+) : $537\left(\mathrm{MH}^{+}\right)$. HRMS for $\mathrm{C}_{28} \mathrm{H}_{34} \mathrm{BrN}_{4} \mathrm{O}_{2}\left(\mathrm{MH}^{+}\right)$: calcd : 537.1860; found : 537.1861. Elem. Anal. for $\mathrm{C}_{28} \mathrm{H}_{33} \mathrm{BrN}_{4} \mathrm{O}_{2}$ : calcd: C, 62.57\%; H, 6.19\%; Br, 14.87, N, 10.42\%; found : C, 62.76\%; H, 6.26\%; Br, 14.82, N, 10.47\%.

N,N',N',N','-Tetramethyl-1-(3-ethylphenylureyl)-3-(2-bromophenylureyl)benzene 9c. -In a similar way 3-nitrophenylisocyanate (1.62 g, $9.90 \mathrm{mmol}, 1.2 \mathrm{eq})$ and 3-ethylaniline (1.02 mL, $8.25 \mathrm{mmol}, 1 \mathrm{eq})$ gave, after the hydrogenation, $1.27 \mathrm{~g}$ of crude material. 2-Bromophenylisocyanate (1.22 $\mathrm{mL}, 9.94$ mmol, 2.0 eq.) was used instead of phenylisocyanate. The methylation was carried out in THF (30 mL) with $\mathrm{NaH} 60 \%$ in mineral oil (996 mg, $24.9 \mathrm{mmol}, 5$ eq.) and methyl iodide (1.55 mL, 24.9 mmol, 5 eq.). After the same treatment, purification by flash chromatography on silica $\left(90 / 10: \mathrm{CH}_{2} \mathrm{Cl}_{2} / \mathrm{MeOH}\right)$ and recrystallised from $\mathrm{Et}_{2} \mathrm{O} /$ petroleum ether gave urea 9c $(1.81 \mathrm{~g}, 43 \%)$ as a white powder. m.p. : $82^{\circ} \mathrm{C} . \mathrm{IR} v_{\max } \mathrm{cm}^{-1}: 2963,2933,1659,1651,1598,1428,1353,1119 .{ }^{1} \mathrm{H}$ NMR $\left(500 \mathrm{MHz}, \mathrm{CDCl}_{3}\right) \delta$ $(\mathrm{ppm}): 1.05(3 \mathrm{H}, \mathrm{t}, J=7.6 \mathrm{~Hz}), 2.38(2 \mathrm{H}, \mathrm{q}, J=7.6 \mathrm{~Hz}), 2.96(3 \mathrm{H}, \mathrm{s}), 2.97(3 \mathrm{H}, \mathrm{s}), 3.08(3 \mathrm{H}, \mathrm{s}), 3.13$ 
$(3 \mathrm{H}, \mathrm{s}), 6.05(1 \mathrm{H}, \mathrm{t}, J=2.0 \mathrm{~Hz}), 6.43(2 \mathrm{H}, \mathrm{m}), 6.46(1 \mathrm{H}, \mathrm{s}), 6.56(1 \mathrm{H}, \mathrm{dd}, J=1.2,7.9 \mathrm{~Hz}), 6.64(1 \mathrm{H}$, $\mathrm{dd}, J=1.5,7.9 \mathrm{~Hz}), 6.71(1 \mathrm{H}, \mathrm{d}, J=8.0 \mathrm{~Hz}), 6.75(1 \mathrm{H}, \mathrm{t}, J=8.0 \mathrm{~Hz}), 6.80(1 \mathrm{H}, \mathrm{dt}, J=1.6,7.9 \mathrm{~Hz})$, $6.90(2 \mathrm{H}, \mathrm{m}), 7.27(1 \mathrm{H}, \mathrm{dd}, J=1.2,8.0 \mathrm{~Hz}) .{ }^{13} \mathrm{C} \mathrm{NMR}\left(75 \mathrm{MHz}, \mathrm{CDCl}_{3}\right) \delta(\mathrm{ppm}): 15.4,28.4,38.5$, $39.0,39.4,39.6,122.5,122.6,122.8,123.0$ (2C), 124.5, 125.2 (2C), 127.3, 127.7, 128.4, 128.6, 129.9, 133.0, 143.6, 144.7, 145.3, 145.9, 160.2, 160.8. MS (ESI+) : $509\left(\mathrm{MH}^{+}\right)$. HRMS for $\mathrm{C}_{26} \mathrm{H}_{30} \mathrm{BrN}_{4} \mathrm{O}_{2}$ $\left(\mathrm{MH}^{+}\right)$: calcd : 509.1547; found : 509.1552. Elem. Anal. for $\mathrm{C}_{26} \mathrm{H}_{29} \mathrm{BrN}_{4} \mathrm{O}_{2}$ : calcd: C, 61.30\%; H, 5.74\%; Br, 15.68, N, 11.00\%; found : C, 60.88\%; H, 5.55\%; Br, 16.09, N, 10.93\%.

$N, N$, $N$ ', $N$ ',-Tetramethyl-1-(3-isopropylphenylureyl)-3-(2-bromophenylureyl)benzene 9d. -In a similar way, 3-nitrophenylisocyanate $(980 \mathrm{mg}, 5.97 \mathrm{mmol}, 1.0$ eq.) and 3-isopropylaniline (1.01 mL, $7.16 \mathrm{mmol}, 1.2 \mathrm{eq})$ gave, after the hydrogenation, $1.61 \mathrm{~g}$ of crude material. 2-Bromophenylisocyanate (1.48 mL, $12.0 \mathrm{mmol}, 2.0$ eq.) was used instead of phenylisocyanate. The methylation was carried out in THF (40 mL) with NaH 60\% in mineral oil (1.19 g, 29.9 mmol, 5 eq.) and methyl iodide (1.86 mL, 29.9 mmol, 5 eq.). Recrystallisation from $\mathrm{Et}_{2} \mathrm{O} /$ petroleum ether gave urea 9d $(2.09 \mathrm{~g}, 67 \%)$ as a white powder. m.p. : $106^{\circ} \mathrm{C}$. IR $v_{\max } \mathrm{cm}^{-1}: 2960,2926,1659,1599,1429,1354,1119 .{ }^{1} \mathrm{H}$ NMR $(500 \mathrm{MHz}$, $\left.\mathrm{CDCl}_{3}\right) \delta(\mathrm{ppm}): 1.05(6 \mathrm{H}, \mathrm{d}, J=7.0 \mathrm{~Hz}), 2.62(1 \mathrm{H}, \mathrm{sept}, J=7.0 \mathrm{~Hz}), 2.96(3 \mathrm{H}, \mathrm{s}), 2.97(3 \mathrm{H}, \mathrm{s}), 3.08$ $(3 \mathrm{H}, \mathrm{s}), 3.13(3 \mathrm{H}, \mathrm{s}), 6.04(1 \mathrm{H}, \mathrm{t}, J=2.0 \mathrm{~Hz}), 6.42,(2 \mathrm{H}, \mathrm{dd}, J=2.0,8.0 \mathrm{~Hz}), 6.48(1 \mathrm{H}, \mathrm{t}, J=1.7 \mathrm{~Hz})$, $6.55(1 \mathrm{H}, J=7.9 \mathrm{~Hz}), 6.64(1 \mathrm{H}, \mathrm{dd}, J=1.6,7.9 \mathrm{~Hz}), 6.74(2 \mathrm{H}, \mathrm{m}), 6.79(1 \mathrm{H}, \mathrm{dt}, J=1.6,7.8 \mathrm{~Hz}), 6.89$ $(1 \mathrm{H}, \mathrm{dt}, J=1.4,7.7 \mathrm{~Hz}), 6.92(1 \mathrm{H}, \mathrm{t}, J=7.8 \mathrm{~Hz}), 7.27(1 \mathrm{H}, \mathrm{dd}, J=1.5,8.0 \mathrm{~Hz}) .{ }^{13} \mathrm{C} \mathrm{NMR}(75 \mathrm{MHz}$, $\left.\mathrm{CDCl}_{3}\right) \delta(\mathrm{ppm}): 23.8(2 \mathrm{C}), 33.7,38.5,39.0,39.4,39.6,122.5,122.6,122.8,123.0,123.1(2 \mathrm{C}), 123.8$, $127.3,127.7,128.3,128.6,129.9,133.0,143.6,145.2,145.3,145.9,149.4,160.1,160.8$. MS (ESI+) : $523\left(\mathrm{MH}^{+}, 10\right), 525\left(\mathrm{MH}^{+}, 15\right), 545(\mathrm{M}+\mathrm{Na}, 100), 547(\mathrm{M}+\mathrm{Na}, 85)$. HRMS for $\mathrm{C}_{27} \mathrm{H}_{31} \mathrm{BrN}_{4} \mathrm{O}_{2} \mathrm{Na}$ $(\mathrm{M}+\mathrm{Na})$ : calcd : 545.1523; found : 545.1520. Elem. Anal. for $\mathrm{C}_{27} \mathrm{H}_{31} \mathrm{BrN}_{4} \mathrm{O}_{2}$ : calcd: $\mathrm{C}, 61.95 \%$; $\mathrm{H}$, 5.97\%; Br, 15.26, N, 10.70\%; found : C, 61.79\%; H, 5.93\%; Br, 15.46, N, $10.65 \%$.

\section{$N, N$, $N$ ',,$N$ ',,$N$,',,$N$,',',-Hexamethyl-1-[3-(2-bromophenylureyl)phenyl]-3-[3-(2,6-}


diethylphenylureyl)phenyl]urea 10b. - By the method used for 10a, 3-nitrophenylisocyanate (300 mg, $1.83 \mathrm{mmol}, 1$ eq.), 2,6-diethylaniline (361 $\mu \mathrm{L}, 2.19 \mathrm{mmol}, 1.2 \mathrm{eq})$ and finally 2-bromophenylisocyanate (464 $\mu \mathrm{L}, 2.34 \mathrm{mmol}, 2.0$ eq.) gave, after methylation in THF (40 mL) with $\mathrm{NaH} 60 \%$ in mineral oil (374 $\mathrm{mg}, 9.36 \mathrm{mmol}, 8$ eq.) and methyl iodide (583 $\mu \mathrm{L}, 9.36 \mathrm{mmol}, 8$ eq.), and purification by flash chromatography on silica gel $\left(\mathrm{CH}_{2} \mathrm{Cl}_{2} / \mathrm{MeOH}: 98 / 2\right)$ the urea $\mathbf{1 0 b}(539 \mathrm{mg}, 42 \%)$ as a white powder. m.p. : $148^{\circ} \mathrm{C}$. IR $v_{\max } \mathrm{cm}^{-1}: 2967,2933,1660,1651,1598,1429,1353,1107 .{ }^{1} \mathrm{H}$ NMR $(500 \mathrm{MHz}$, $\left.\mathrm{CDCl}_{3}\right) \delta(\mathrm{ppm}): 1.04(6 \mathrm{H}, \mathrm{t}, J=7.6 \mathrm{~Hz}), 2.07(2 \mathrm{H}, \mathrm{dq}, J=15.1,7.6 \mathrm{~Hz}), 2.28(2 \mathrm{H}, \mathrm{dq}, J=15.1,7.5$ Hz), $2.77(3 \mathrm{H}, \mathrm{s}), 2.90(3 \mathrm{H}, \mathrm{s}), 2.92(6 \mathrm{H}, \mathrm{s}), 3.01(3 \mathrm{H}, \mathrm{s}), 3.07(3 \mathrm{H}, \mathrm{s}), 5.79(1 \mathrm{H}, \mathrm{s}), 6.03(1 \mathrm{H}, \mathrm{s}), 6.28$ $(1 \mathrm{H}, \mathrm{d}, J=7.7 \mathrm{~Hz}), 6.33(1 \mathrm{H}, \mathrm{d}, J=7.8 \mathrm{~Hz}), 6.38(1 \mathrm{H}, \mathrm{d}, J=8.0 \mathrm{~Hz}), 6.42(1 \mathrm{H}, \mathrm{d}, J=7.9 \mathrm{~Hz}), 6.63$ $(1 \mathrm{H}, \mathrm{d}, J=7.8 \mathrm{~Hz}), 6.71(2 \mathrm{H}, \mathrm{t}, J=7.9 \mathrm{~Hz}), 6.77(2 \mathrm{H}, \mathrm{d}, J=7.5 \mathrm{~Hz}), 6.79(1 \mathrm{H}, \mathrm{t}, J=7.7 \mathrm{~Hz}), 6.88$ $(1 \mathrm{H}, \mathrm{t}, J=7.6 \mathrm{~Hz}), 6.94(1 \mathrm{H}, \mathrm{t}, J=7.6 \mathrm{~Hz}), 7.27(1 \mathrm{H}, \mathrm{d}, J=7.7 \mathrm{~Hz}) .{ }^{13} \mathrm{C} \mathrm{NMR}\left(125 \mathrm{MHz}, \mathrm{CDCl}_{3}\right) \delta$ (ppm) : 14.1 (2C), 23.3 (2C), 38.3, 38.5, 39.0 (2C), 39.6, 40.8, 122.3, 122.5, 122.6, 122.7, 122.8, 123.0, $123.3,125.7,127.3,127.4,127.7,128.4,128.6,129.8,133.1,140.6,141.3,143.6,145.4,145.5,145.6$, 145.7, 160.1, 160.4, 161.3. MS (ESI+) : $721(\mathrm{M}+\mathrm{Na}, 90), 723(\mathrm{M}+\mathrm{Na}, 100)$. HRMS for $\mathrm{C}_{37} \mathrm{H}_{43} \mathrm{~N}_{6} \mathrm{O}_{3} \mathrm{BrNa}(\mathrm{M}+\mathrm{Na})$ : calcd : 721.2472; found : 721.2472. Elem. Anal. for $\mathrm{C}_{37} \mathrm{H}_{43} \mathrm{~N}_{6} \mathrm{O}_{3} \mathrm{Br}$ : calcd: C, 63.51\%; H, 6.19\%; Br, 11.42\%; N, 12.01\%; found : C, 63.32\%; H, 6.23\%; Br, 11.17\%; N, $11.99 \%$.

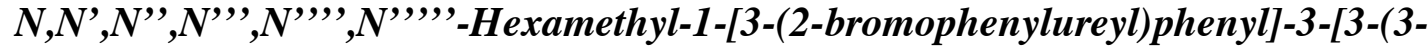

isopropylphenylureyl)phenyl]urea 10c. - In a similar way, 3-nitrophenylisocyanate (500 mg, 3.05 mmol, 1 eq.), 3-isopropylaniline (518 $\mu \mathrm{L}, 3.66 \mathrm{mmol}, 1.2 \mathrm{eq})$ and finally 2-bromophenylisocyanate (611 $\mu \mathrm{L}, 4.96 \mathrm{mmol}, 2.0$ eq.) gave, after methylation in THF (50 mL) with $\mathrm{NaH} 60 \%$ in mineral oil $(532 \mathrm{mg}$, $13.3 \mathrm{mmol}, 8$ eq.) and methyl iodide (827 $\mu \mathrm{L}, 13.3 \mathrm{mmol}, 8$ eq.) and purification by flash chromatography on silica gel $\left(\mathrm{CH}_{2} \mathrm{Cl}_{2} / \mathrm{MeOH}: 98 / 2\right)$, the urea 10c $(853 \mathrm{mg}, 41 \%)$ as a white powder. m.p. : $111^{\circ} \mathrm{C}$. IR $v_{\max } \mathrm{cm}^{-1}: 2960,2930,1660,1598,1429,1354,1117 .{ }^{1} \mathrm{H}$ NMR $\left(500 \mathrm{MHz}, \mathrm{CDCl}_{3}\right) \delta$ (ppm) : $1.05(6 \mathrm{H}, \mathrm{d}, J=6.9 \mathrm{~Hz}), 2.62(1 \mathrm{H}, \mathrm{sept}, J=6.9 \mathrm{~Hz}), 2.92(6 \mathrm{H}, \mathrm{s}), 2.93(3 \mathrm{H}, \mathrm{s}), 2.94(3 \mathrm{H}, \mathrm{s})$, 
$3.07(3 \mathrm{H}, \mathrm{s}), 3.12(3 \mathrm{H}, \mathrm{s}), 6.02(1 \mathrm{H}, \mathrm{t}, J=2.1 \mathrm{~Hz}), 6.05(1 \mathrm{H}, \mathrm{t}, J=2.0 \mathrm{~Hz}), 6.37(4 \mathrm{H}, \mathrm{m}), 6.46(1 \mathrm{H}, \mathrm{s})$, $6.52(1 \mathrm{H}, \mathrm{d}, J=7.9 \mathrm{~Hz}), 6.64(1 \mathrm{H}, \mathrm{d}, J=7.9 \mathrm{~Hz}), 6.70(1 \mathrm{H}, \mathrm{t}, J=8.0 \mathrm{~Hz}), 6.72(1 \mathrm{H}, \mathrm{t}, J=8.0 \mathrm{~Hz})$, $6.74(1 \mathrm{H}, \mathrm{d}, J=7.6 \mathrm{~Hz}), 6.79(1 \mathrm{H}, \mathrm{dt}, J=1.6,7.4 \mathrm{~Hz}), 6.89(2 \mathrm{H}, \mathrm{m}), 7.27(1 \mathrm{H}, \mathrm{d}, J=7.9 \mathrm{~Hz}) .{ }^{13} \mathrm{C}$ NMR (125 MHz, $\left.\mathrm{CDCl}_{3}\right) \delta(\mathrm{ppm}): 23.8$ (2C), 33.7, 38.5, 39.06, 39.09, 39.11, 39.4, 39.7, 122.1, 122.3, $122.5,122.6,122.9,123.0,123.1$ (3C), 123.8, 127.4, 127.7, 128.3, 128.3, 128.7, 129.8, 133.1, 143.6, 145.3, 145.4, 145.5, 145.7, 145.8, 149.4, 160.1, 160.4, 160.7. MS (ESI+) : $685\left(\mathrm{MH}^{+}, 95\right), 687\left(\mathrm{MH}^{+}\right.$ 100). HRMS for $\mathrm{C}_{36} \mathrm{H}_{42} \mathrm{~N}_{6} \mathrm{O}_{3} \mathrm{Br}\left(\mathrm{MH}^{+}\right)$: calcd : 685.2496; found : 685.2489. Elem. Anal. for $\mathrm{C}_{36} \mathrm{H}_{41} \mathrm{~N}_{6} \mathrm{O}_{3} \mathrm{Br}$ : calcd: C, 63.06\%; H, 6.03\%; Br, 11.65\%; N, 12.26\%; found : C, 62.61\%; H, 6.05\%; $\mathrm{Br}$, $12.05 \% ; \mathrm{N}, 12.26 \%$.

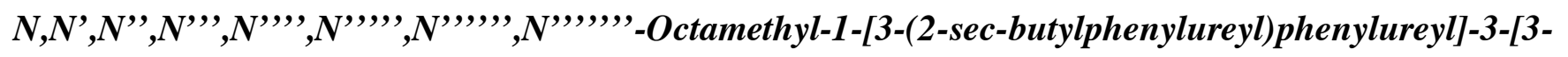
(2-isopropylphenylureyl)phenylureyl]benzene 11b. - By the method used for 11a, 3nitrophenylisocyanate $(600 \mathrm{mg}, 3.66 \mathrm{mmol}, 1$ eq.) and 2-isopropylphenylisocyanate (760 $\mu \mathrm{L}, 4.80$ mmol, 2.0 eq.) gave, after methylation in THF (50 mL) with $\mathrm{NaH} 60 \%$ in mineral oil (960 mg, 24.0 mmol, 10 eq.) and methyl iodide $(1.50 \mathrm{~mL}, 24.0 \mathrm{mmol}, 10$ eq. $)$ and purification by flash chromatography on silica gel $\left(\mathrm{CH}_{2} \mathrm{Cl}_{2} / \mathrm{MeOH}: 90 / 10\right)$ the product $11 \mathbf{b}(1.06 \mathrm{~g}, 35 \%)$ as a white powder. m.p. : $80-86^{\circ} \mathrm{C}$. IR $v_{\max } \mathrm{cm}^{-1}: 3004,2964,2939,1659,1650,1598,1429,1354,873 .{ }^{1} \mathrm{H}$ NMR $\left(500 \mathrm{MHz}, \mathrm{CDCl}_{3}\right) \delta(\mathrm{ppm}): 0.68-1.46(8 \mathrm{H}, \mathrm{bm}), 1.08(6 \mathrm{H}, \mathrm{d}, J=6.7 \mathrm{~Hz}), 2.49(1 \mathrm{H}, \mathrm{sext}, J=7.0 \mathrm{~Hz})$ $2.78(1 \mathrm{H}, \mathrm{m}), 2.79(3 \mathrm{H}, \mathrm{s}), 2.80(3 \mathrm{H}, \mathrm{s}), 2.88(6 \mathrm{H}, \mathrm{s}), 2.90(6 \mathrm{H}, \mathrm{s}), 3.05(3 \mathrm{H}, \mathrm{s}), 3.06(3 \mathrm{H}, \mathrm{s}), 5.90(1 \mathrm{H}$, $\mathrm{t}, J=2.0 \mathrm{~Hz}), 5.91(1 \mathrm{H}, \mathrm{t}, J=2.0 \mathrm{~Hz}), 6.02(1 \mathrm{H}, \mathrm{t}, J=2.0 \mathrm{~Hz}), 6.19(1 \mathrm{H}, \mathrm{d}, J=7.7 \mathrm{~Hz}), 6.26(1 \mathrm{H}, \mathrm{dd}$, $J=1.0,7.9 \mathrm{~Hz}), 6.30(2 \mathrm{H}, \mathrm{d}, J=8.0 \mathrm{~Hz}), 6.34(2 \mathrm{H}, \mathrm{d}, J=7.9 \mathrm{~Hz}), 6.38(2 \mathrm{H}, \mathrm{d}, J=8.0 \mathrm{~Hz}), 6.59(1 \mathrm{H}$, $\mathrm{t}, J=7.2 \mathrm{~Hz}), 6.62(1 \mathrm{H}, \mathrm{t}, J=7.8 \mathrm{~Hz}), 6.67(1 \mathrm{H}, \mathrm{t}, J=8.0 \mathrm{~Hz}), 6.73(2 \mathrm{H}, \mathrm{t}, J=8.0 \mathrm{~Hz}), 6.93(2 \mathrm{H}, \mathrm{m})$, $7.02(1 \mathrm{H}, \mathrm{dd}, J=1.4,7.8 \mathrm{~Hz}), 7.07(1 \mathrm{H}, \mathrm{dd}, J=1.4,7.9 \mathrm{~Hz}) .{ }^{13} \mathrm{C} \mathrm{NMR}\left(125 \mathrm{MHz}, \mathrm{CDCl}_{3}\right) \delta(\mathrm{ppm}):$ $12.5,21.0,23.0,25.28,27.2,31.9,34.4,39.0(2 \mathrm{C}), 39.1(2 \mathrm{C}), 39.69,39.71,40.0(2 \mathrm{C}), 122.0,122.48$ $122.50,122.6,123.2,125.7,125.8,126.26,126.3,126.7,126.8,128.3,128.4,128.6,128.7,141.8,145.6$ (2C), 145.8 (2C), 145.9, 146.5 (2C), 146.6, 160.3 (2C), 161.6 (2C). MS (ESI+) : 847 (M+Na). HRMS 
for $\mathrm{C}_{49} \mathrm{H}_{60} \mathrm{~N}_{8} \mathrm{O}_{4} \mathrm{Na}(\mathrm{M}+\mathrm{Na})$ : calcd : 847.4630; found : 847.4622. Elem. Anal. for $\mathrm{C}_{49} \mathrm{H}_{60} \mathrm{~N}_{8} \mathrm{O}_{4}$ : calcd: C, 71.33\%; H, 7.33\%; N, 13.58\%; found : C, 70.91\%; H, 7.45\%; N, $13.44 \%$.

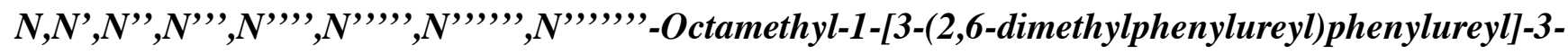

[3-(2-bromophenylureyl)phenylureyl]benzene 11c. - In a similar way, 3-nitrophenylisocyanate (300 $\mathrm{mg}, \quad 1.83 \mathrm{mmol}, 1$ eq. $),$ 2,6-diethylaniline $(360 \mu \mathrm{L}, 2.19 \mathrm{mmol}, 1.2 \mathrm{eq})$ and finally 2bromophenylisocyanate $(215 \mu \mathrm{L}, 1.74 \mathrm{mmol}, 2.0$ eq.) gave, after methylation in THF (20 mL) with $\mathrm{NaH}$ $60 \%$ in mineral oil ( $348 \mathrm{mg}, 8.71 \mathrm{mmol}, 10$ eq.) and methyl iodide (542 $\mu \mathrm{L}, 8.71 \mathrm{mmol}, 10$ eq.) and purification by flash chromatography on silica gel $\left(\mathrm{CH}_{2} \mathrm{Cl}_{2} / \mathrm{MeOH}: 98 / 2\right)$ the urea $11 \mathrm{c}(273 \mathrm{mg}, 17 \%)$ as a white powder. m.p. : $176^{\circ} \mathrm{C}$. IR $v_{\max } \mathrm{cm}^{-1}: 3054,2967,1659,1650,1428,1352,1108 .{ }^{1} \mathrm{H}$ NMR $\left(500 \mathrm{MHz}, \mathrm{CDCl}_{3}\right) \delta(\mathrm{ppm}): 1.04(6 \mathrm{H}, \mathrm{t}, J=7.5 \mathrm{~Hz}), 2.07(2 \mathrm{H}, \mathrm{dq}, J=15.1,7.5 \mathrm{~Hz}), 2.28(2 \mathrm{H}, \mathrm{dq}, J=$ 15.1, $7.5 \mathrm{~Hz}), 2.77(3 \mathrm{H}, \mathrm{s}), 2.89(9 \mathrm{H}, \mathrm{s}), 2.92_{5}(3 \mathrm{H}, \mathrm{s}), 2.93_{1}(3 \mathrm{H}, \mathrm{s}), 3.02(3 \mathrm{H}, \mathrm{s}), 3.08(3 \mathrm{H}, \mathrm{s}), 5.79(1 \mathrm{H}$ s), $6.00(1 \mathrm{H}, \mathrm{s}), 6.05(1 \mathrm{H}, \mathrm{s}), 6.31(4 \mathrm{H}, \mathrm{m}), 6.40(2 \mathrm{H}, \mathrm{t}, J=6.4 \mathrm{~Hz}), 6.68(4 \mathrm{H}, \mathrm{m}), 6.79(3 \mathrm{H}, \mathrm{m}), 6.89$ $(1 \mathrm{H}, \mathrm{dt}, J=1.5,7.8 \mathrm{~Hz}), 6.94(1 \mathrm{H}, \mathrm{t}, J=7.5 \mathrm{~Hz}), 7.28(1 \mathrm{H}, \mathrm{dd}, J=1.4,8.0 \mathrm{~Hz}) .{ }^{13} \mathrm{C} \mathrm{NMR}(125 \mathrm{MHz}$, $\left.\mathrm{CDCl}_{3}\right) \delta(\mathrm{ppm}): 14.1(2 \mathrm{C}), 23.4(2 \mathrm{C}), 38.3,38.4,38.5,39.03,39.05,39.08,39.10,39.7,122.0,122.1$, $122.48,122.52,122.6,122.7,122.8,122.9,123.00,123.3,125.7,127.3,127.4,127.8,128.2,128.3$, 128.40, 128.41, 129.8, 133.1, 160.1, 160.2, 160.3, 161.4. MS (ESI+) : 883, 885 (M+Na, 100). HRMS for $\mathrm{C}_{46} \mathrm{H}_{53} \mathrm{BrN}_{8} \mathrm{O}_{4} \mathrm{Na}(\mathrm{M}+\mathrm{Na})$ : calcd : 883.3265; found : 883.3274.

\section{$N, N, N,, N, N^{,,}, N,,,, N,,,,, N,,,,,, N,,,,,, '$ Octamethyl-1-[3-(2-isopropylphenylureyl)phenylureyl]-3-}

[3-(2-bromophenylureyl)phenylureyl]benzene 11d. - In a similar way, 3-nitrophenylisocyanate (700 $\mathrm{mg}, 4.27 \mathrm{mmol}, 1$ eq.), 2-isopropylaniline (725 $\mu \mathrm{L}, 5.12 \mathrm{mmol}, 1.2 \mathrm{eq})$ and finally 2bromophenylisocyanate $(1.00 \mathrm{~mL}, 8.09 \mathrm{mmol}, 2.0$ eq.) gave, after methylation in $\mathrm{THF}(50 \mathrm{~mL})$ with $\mathrm{NaH} 60 \%$ in mineral oil (1.62 g, $40.5 \mathrm{mmol}, 10$ eq.) and methyl iodide (2.50 mL, $40.5 \mathrm{mmol}, 10$ eq.) and purification by flash chromatography on silica gel (AcOEt), the urea 11d (623 mg, 17\%) was isolated as a white powder. m.p. : $122^{\circ} \mathrm{C}$. IR $v_{\max } \mathrm{cm}^{-1}: 3072,1659,1650,1598,1428,1353 .{ }^{1} \mathrm{H}$ NMR 
$\left(500 \mathrm{MHz}, \mathrm{CDCl}_{3}\right) \delta(\mathrm{ppm}): 1.07(6 \mathrm{H}, \mathrm{d}, J=6.5 \mathrm{~Hz}), 2.78(1 \mathrm{H}, \mathrm{m}), 2.80(3 \mathrm{H}, \mathrm{s}), 2.88(3 \mathrm{H}, \mathrm{s}), 2.89$ $(3 \mathrm{H}, \mathrm{s}), 2.90(3 \mathrm{H}, \mathrm{s}), 2.91(3 \mathrm{H}, \mathrm{s}), 2.92(3 \mathrm{H}, \mathrm{s}), 3.05(3 \mathrm{H}, \mathrm{s}), 3.06(3 \mathrm{H}, \mathrm{s}), 5.91(1 \mathrm{H}, \mathrm{t}, J=2.0 \mathrm{~Hz}), 6.02$ $(1 \mathrm{H}, \mathrm{t}, J=2.0 \mathrm{~Hz}), 6.04(1 \mathrm{H}, \mathrm{t}, J=2.0 \mathrm{~Hz}), 6.26(1 \mathrm{H}, \mathrm{dd}, J=1.5,8.0 \mathrm{~Hz}), 6.32(4 \mathrm{H}, \mathrm{m}), 6.39(2 \mathrm{H}, \mathrm{m})$, $6.64(2 \mathrm{H}, \mathrm{m}), 6.68(1 \mathrm{H}, \mathrm{t}, J=8.0 \mathrm{~Hz}), 6.70(1 \mathrm{H}, \mathrm{t}, J=8.0 \mathrm{~Hz}), 6.73(1 \mathrm{H}, \mathrm{t}, J=8.0 \mathrm{~Hz}), 6.79(1 \mathrm{H}, \mathrm{dt}, J$ $=1.2,7.5 \mathrm{~Hz}), 6.88(1 \mathrm{H}, \mathrm{dt}, J=1.5,7.5 \mathrm{~Hz}), 6.94(1 \mathrm{H}, \mathrm{dt}, J=1.2,7.5 \mathrm{~Hz}), 7.07(1 \mathrm{H}, \mathrm{dd}, J=1.5,7.8$ $\mathrm{Hz}), 7.26(1 \mathrm{H}, \mathrm{dd}, J=1.5,8.0 \mathrm{~Hz}) .{ }^{13} \mathrm{C} \mathrm{NMR}\left(75 \mathrm{MHz}, \mathrm{CDCl}_{3}\right) \delta(\mathrm{ppm}): 22.0,27.2,38.5,39.08,39.10$ (2C), 39.2, 39.7, 39.8, 40.0, 122.0, 122.1, 122.49, 122.52, 122.54 (2C), 122.8, 123.20, 125.8, 126.3, $126.8,127.4,127.8,128.4,128.5,128.67,128.72,129.8,133.1,141.8,143.6,145.4(2 \mathrm{C}), 145.60(2 \mathrm{C})$, 145.6, 145.7, 145.8, 146.5, 160.1, 160.2, 160.3, 161.6. MS (APCI+) : $847\left(\mathrm{MH}^{+}\right)$. HRMS for $\mathrm{C}_{45} \mathrm{H}_{52} \mathrm{BrN}_{8} \mathrm{O}_{4}\left(\mathrm{MH}^{+}\right)$: calcd : 847.3289; found : 847.3286. Elem. Anal. for $\mathrm{C}_{45} \mathrm{H}_{51} \mathrm{BrN}_{8} \mathrm{O}_{4}$ : calcd: C, 63.75\%; H, 6.06\%; Br, 9.42\%; N, 13.22\%; found : C, 64.02\%; H, 6.17\%; Br, 9.33\%; N, $13.06 \%$.

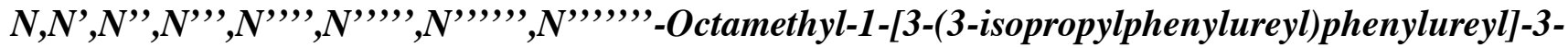

[3-(2-bromophenylureyl)phenylureyl]benzene 11e. - In a similar way, 3-nitrophenylisocyanate (550 mg, 3.35 mmol, 1 eq.), 3-isopropylaniline $(570 \mu \mathrm{L}, 4.02 \mathrm{mmol}, 1.2 \mathrm{eq})$ and finally 2bromophenylisocyanate $(460 \mu \mathrm{L}, 3.73 \mathrm{mmol}, 2.0$ eq.) gave, after methylation in THF $(50 \mathrm{~mL})$ with $\mathrm{NaH} 60 \%$ in mineral oil ( $581 \mathrm{mg}, 14.5 \mathrm{mmol}, 10$ eq.) and methyl iodide (903 $\mu \mathrm{L}, 14.5 \mathrm{mmol}, 10$ eq.) and purification by flash chromatography on silica gel $\left(\mathrm{CH}_{2} \mathrm{Cl}_{2} / \mathrm{MeOH}: 98 / 2\right)$, the urea $11 \mathrm{e}(833 \mathrm{mg}$, $30 \%$ ) as a white powder. m.p. : $136^{\circ} \mathrm{C}$. IR $v_{\max } \mathrm{cm}^{-1}: 3055,2961,1659,1598,1429,1353,1108 .{ }^{1} \mathrm{H}$ $\operatorname{NMR}\left(500 \mathrm{MHz}, \mathrm{CDCl}_{3}\right) \delta(\mathrm{ppm}): 1.04(6 \mathrm{H}, \mathrm{d}, J=7.0 \mathrm{~Hz}), 2.62(1 \mathrm{H}$, sept,$J=7.0), 2.88(3 \mathrm{H}, \mathrm{s}), 2.90$ $(3 \mathrm{H}, \mathrm{s}), 2.91(6 \mathrm{H}, \mathrm{s}), 2.92(3 \mathrm{H}, \mathrm{s}), 2.93(3 \mathrm{H}, \mathrm{s}), 3.07(3 \mathrm{H}, \mathrm{s}), 3.12(3 \mathrm{H}, \mathrm{s}), 6.01(1 \mathrm{H}, \mathrm{t}, J=2.0 \mathrm{~Hz}), 6.02$ $(1 \mathrm{H}, \mathrm{t}, J=2.0 \mathrm{~Hz}), 6.05(1 \mathrm{H}, \mathrm{t}, J=2.0 \mathrm{~Hz}), 6.32(4 \mathrm{H}, \mathrm{m}), 6.39(2 \mathrm{H}, \mathrm{m}), 6.48(1 \mathrm{H}, \mathrm{t}, J=1.8 \mathrm{~Hz}), 6.52$ $(1 \mathrm{H}, \mathrm{d}, J=7.5 \mathrm{~Hz}), 6.64(1 \mathrm{H}, \mathrm{dd}, J=1.6,7.9 \mathrm{~Hz}), 6.70(4 \mathrm{H}, \mathrm{m}), 6.79(1 \mathrm{H}, \mathrm{t}, J=7.9 \mathrm{~Hz}), 6.89(2 \mathrm{H}, \mathrm{m})$, $7.27(1 \mathrm{H}, \mathrm{dd}, J=1.4,7.9 \mathrm{~Hz}) .{ }^{13} \mathrm{C} \mathrm{NMR}\left(125 \mathrm{MHz}, \mathrm{CDCl}_{3}\right) \delta(\mathrm{ppm}): 23.8(2 \mathrm{C}), 33.7,38.5,39.0,39.09$ 39.12 (3C), 39.4, 39.6, 122.01, 122.03, 122.11, 122.3, 122.4, 122.5, 122.6, 122.8, 122.9, 123.0, 123.1, $123.2,123.8,127.4,127.7,128.3,128.4(2 \mathrm{C}), 128.7,129.8,133.1,143.6,145.3,145.4(2 \mathrm{C}), 145.6$, 
145.6, 145.7, 145.9, 149.4, 160.1, 160.2, 160.3, 160.8. MS (ESI+) : $869(\mathrm{M}+\mathrm{Na}, 100), 871(\mathrm{M}+\mathrm{Na}$, 100). HRMS for $\mathrm{C}_{45} \mathrm{H}_{51} \mathrm{BrN}_{8} \mathrm{O}_{4} \mathrm{Na}(\mathrm{M}+\mathrm{Na})$ : calcd : 869.3109; found : 869.3116. Elem. Anal. for $\mathrm{C}_{45} \mathrm{H}_{51} \mathrm{BrN}_{8} \mathrm{O}_{4}$ : calcd: C, 63.75\%; H, 6.06\%; Br, 9.42\%; N, 13.22\%; found : C, 63.50\%; H, 6.19\%; $\mathrm{Br}$, $9.83 \% ; \mathrm{N}, 13.09 \%$.

N,N','Dibenzyl-N',N'’dimethyl-1,3-di-(2-bromophenylureyl)benzene 16. -Under a nitrogen atmosphere, 1,3-phenylenediisocyanate (300 mg, $1.87 \mathrm{mmol}, 1$ eq.) was dissolved in dry THF (10 mL) and $N$-benzylaniline $(680 \mu \mathrm{L}, 3.93 \mathrm{mmol}, 2.1 \mathrm{eq})$ was added. The reaction mixture was stirred at r.t. for 3h and evaporated under reduced pressure. $\mathrm{CH}_{2} \mathrm{Cl}_{2}$ was added and the solid was collected by filtration. The white powder was dried under vacuum and suspended in THF (10 mL). NaH $60 \%$ in mineral oil (224 mg, $5.61 \mathrm{mmol}, 3$ eq.) and methyl iodide (350 $\mu \mathrm{L}, 5.61 \mathrm{mmol}, 3$ eq.) were added successively, and the reaction mixture was stirred overnight before adding $\mathrm{H}_{2} \mathrm{O}(2 \mathrm{~mL})$. The aqueous phase was extracted with $\mathrm{CH}_{2} \mathrm{Cl}_{2}$ and the combined organic phases were dried $\left(\mathrm{MgSO}_{4}\right)$ and concentrated under reduced pressure. The crude mixture was washed with $\mathrm{Et}_{2} \mathrm{O}$ and the product $16(628 \mathrm{mg}, 61 \%)$ was collected as a white powder by filtration. m.p. : $164^{\circ} \mathrm{C} . \mathrm{IR} v_{\max } \mathrm{cm}^{-1}: 3069,2980,1649,1596,1432,861 .{ }^{1} \mathrm{H}$ NMR $\left(300 \mathrm{MHz}, \mathrm{CDCl}_{3}\right) \delta(\mathrm{ppm}): 2.91(6 \mathrm{H}, \mathrm{s}), 4.71(4 \mathrm{H}, \mathrm{s}), 5.94(1 \mathrm{H}, \mathrm{t}, J=2.1 \mathrm{~Hz}), 6.37(2 \mathrm{H}, \mathrm{dd}, J=2.2$, $8.0 \mathrm{~Hz}), 6.56(4 \mathrm{H}, \mathrm{dd}, J=1.3,8.0 \mathrm{~Hz}), 6.68(1 \mathrm{H}, \mathrm{t}, J=8.0 \mathrm{~Hz}), 6.89(6 \mathrm{H}, \mathrm{m}), 7.24(10 \mathrm{H}, \mathrm{m}) .{ }^{13} \mathrm{C} \mathrm{NMR}$ $\left(75 \mathrm{MHz}, \mathrm{CDCl}_{3}\right) \delta(\mathrm{ppm}): 39.2,55.2,122.7,123.8,125.1,126.6,127.1,128.2,128.3,128.4,128.7$, 138.3, 144.1, 145.6, 160.6. MS (CI/NH $): 555\left(\mathrm{M}^{+}, 25\right), 346$ (25), 182 (55), 91 (100). HRMS for $\mathrm{C}_{36} \mathrm{H}_{36} \mathrm{~N}_{4} \mathrm{O}_{2}$ : calcd : 556.2833; found : 556.2827 .

$N, N$, $N$ ', $N$ '’-Tetramethyl-1,3-di-(2-isopropylphenylureyl)benzene 17b. - By the method used for 17a, 1,3-phenylenediisocyanate $(500 \mathrm{mg}, 3.12 \mathrm{mmol}, 1 \mathrm{eq})$ and 2-isopropylaniline (920 $\mu \mathrm{L}, 6.55 \mathrm{mmol}$, 2.1 eq) gave, after methylation in THF (30 mL) with $\mathrm{NaH} 60 \%$ in mineral oil $(624 \mathrm{mg}, 15.6 \mathrm{mmol}, 5$ eq.) and methyl iodide $(970 \mu \mathrm{L}, 15.6 \mathrm{mmol}, 5$ eq. $)$, a crude product which was recrystallised from $\mathrm{Et}_{2} \mathrm{O} /$ petroleum ether to yield urea $\mathbf{1 7 b}(1.30 \mathrm{mg}, 86 \%)$ as a white powder. m.p. : $201^{\circ} \mathrm{C}$. IR $v_{\max } \mathrm{cm}^{-1}: 3013$, 
2969, 1650, 1597, 1445. ${ }^{1} \mathrm{H}$ NMR $\left(300 \mathrm{MHz}, \mathrm{CDCl}_{3}\right) \delta(\mathrm{ppm}): 1.10(12 \mathrm{H}, \mathrm{d}, J=6.9 \mathrm{~Hz}), 2.81(2 \mathrm{H}$, sept, $J=6.9 \mathrm{~Hz}), 2.87(6 \mathrm{H}, \mathrm{s}), 3.07(6 \mathrm{H}, \mathrm{s}), 5.88(1 \mathrm{H}, \mathrm{t}, J=2.0 \mathrm{~Hz}), 6.31(2 \mathrm{H}, \mathrm{dd}, J=1.2,7.8 \mathrm{~Hz})$, $6.44(2 \mathrm{H}, \mathrm{dd}, J=2.1,7.5 \mathrm{~Hz}), 6.66(2 \mathrm{H}, \mathrm{dt}, J=1.5,7.5 \mathrm{~Hz}), 6.80(1 \mathrm{H}, \mathrm{t}, J=8.0 \mathrm{~Hz}), 6.95(2 \mathrm{H}, \mathrm{t}, J=$ $7.5 \mathrm{~Hz}), 7.09(2 \mathrm{H}, \mathrm{d}, J=7.8 \mathrm{~Hz}) .{ }^{13} \mathrm{C} \mathrm{NMR}\left(75 \mathrm{MHz}, \mathrm{CDCl}_{3}\right) \delta(\mathrm{ppm}): 22.7,25.2,27.2,39.7,40.1$, $123.1,123.7,125.8,126.2,126.7,128.5,129.0,141.7,145.80,146.7,161.7 . \mathrm{MS}(\mathrm{APCI}+): 487.4$ $\left(\mathrm{MH}^{+}\right)$. HRMS for $\mathrm{C}_{30} \mathrm{H}_{39} \mathrm{~N}_{4} \mathrm{O}_{2}\left(\mathrm{MH}^{+}\right)$: calcd : 487.3068; found : 487.3065. Elem. Anal. for $\mathrm{C}_{30} \mathrm{H}_{38} \mathrm{~N}_{4} \mathrm{O}_{2}$ : calcd: C, 74.04\%; H, 7.87\%; N, 11.51\%; found : C, 74.05\%; H, 7.84\%; N, $11.56 \%$.

$N, N$, $N$ ', $N$ ','-Tetramethyl-1,3-di-(2-ethylphenylureyl)benzene 17c. -In a similar way, 1,3phenylenediisocyanate $(500 \mathrm{mg}, 3.12 \mathrm{mmol}, 1 \mathrm{eq})$ and 2-ethylaniline ( $810 \mu \mathrm{L}, 6.55 \mathrm{mmol}, 2.1 \mathrm{eq})$ gave, after methylation in THF (30 mL) with $\mathrm{NaH} 60 \%$ in mineral oil (624 mg, $15.6 \mathrm{mmol}, 5$ eq.) and methyl iodide ( $970 \mu \mathrm{L}, 15.6 \mathrm{mmol}, 5$ eq.), a crude product which was recrystallised from $\mathrm{Et}_{2} \mathrm{O} /$ petroleum ether to yield urea $17 \mathrm{c}(1.18 \mathrm{mg}, 83 \%)$ as a white powder. m.p. : $128^{\circ} \mathrm{C}$. IR $v_{\max } \mathrm{cm}^{-1}: 3003,2968$, 2933, 1659, 1598, 1355, 877. ${ }^{1} \mathrm{H}$ NMR $\left(300 \mathrm{MHz}, \mathrm{CDCl}_{3}\right) \delta(\mathrm{ppm}): 1.06(6 \mathrm{H}, \mathrm{t}, J=7.5 \mathrm{~Hz}), 2.25(4 \mathrm{H}$, $\mathrm{q}, J=7.5 \mathrm{~Hz}), 2.86(6 \mathrm{H}, \mathrm{s}), 3.03(6 \mathrm{H}, \mathrm{s}), 5.81(1 \mathrm{H}, \mathrm{t}, J=1.7 \mathrm{~Hz}), 6.41(2 \mathrm{H}, \mathrm{dd}, J=2.1,7.8 \mathrm{~Hz}), 6.50$ $(2 \mathrm{H}, \mathrm{d}, J=7.5 \mathrm{~Hz}), 6.77(3 \mathrm{H}, \mathrm{m}), 6.95(4 \mathrm{H}, \mathrm{m}) .{ }^{13} \mathrm{C} \mathrm{NMR}\left(75 \mathrm{MHz}, \mathrm{CDCl}_{3}\right) \delta(\mathrm{ppm}): 13.8,22.9,39.2$, $39.8,123.0,123.5,126.0,126.5,128.0,128.2,128.5,140.5,143.0,146.0,161.4 . \mathrm{MS}(\mathrm{APCI}+): 459.5$ $\left(\mathrm{MH}^{+}\right)$. HRMS for $\mathrm{C}_{28} \mathrm{H}_{35} \mathrm{~N}_{4} \mathrm{O}_{2}\left(\mathrm{MH}^{+}\right)$: calcd : 459.2755; found : 459.2758. Elem. Anal. for $\mathrm{C}_{28} \mathrm{H}_{34} \mathrm{~N}_{4} \mathrm{O}_{2}$ : calcd: C, 73.33\%; H, 7.47\%; N, 12.22\%; found : C, 73.46\%; H, 7.52\%; N, $12.05 \%$.

$N, N$,N', $N$ ','-Tetramethyl-1,3-di-(2-bromophenylureyl)benzene 17d. - Under nitrogen atmosphere, 1,3-phenylenediisocyanate (400 mg, $2.50 \mathrm{mmol}, 1$ eq.) was dissolved in dry THF (10 mL) and 2bromoaniline $(570 \mu \mathrm{L}, 5.25 \mathrm{mmol}, 2.1 \mathrm{eq})$ was added. The reaction mixture was stirred at r.t. for $3 \mathrm{~h}$ and evaporated under reduced pressure. $\mathrm{CH}_{2} \mathrm{Cl}_{2}$ was added and the solid collected by filtration $(1.0 \mathrm{~g})$. The white powder was dried under vacuum and suspended in THF (30 mL). NaOH (408 mg, $10.2 \mathrm{mmol}, 5$ eq.) and methyl iodide (635 $\mu \mathrm{L}, 10.2 \mathrm{mmol}, 5$ eq.) were added successively, and the reaction mixture 
was stirred overnight before adding $\mathrm{H}_{2} \mathrm{O}(2 \mathrm{~mL})$. The aqueous phase was extracted with $\mathrm{CH}_{2} \mathrm{Cl}_{2}$ and the combined organic phases were dried $\left(\mathrm{MgSO}_{4}\right)$ and concentrated under reduced pressure. Flash chromatography on silica gel (AcOEt / petroleum ether : 70/30) afforded $644 \mathrm{mg}(46 \%)$ of product 17d as a white powder. m.p. : $171^{\circ} \mathrm{C}$. IR $v_{\max } \mathrm{cm}^{-1}: 3064,2969,1650,1598,1430,876 .{ }^{1} \mathrm{H} \mathrm{NMR}(300 \mathrm{MHz}$, $\left.\mathrm{CDCl}_{3}\right) \delta(\mathrm{ppm}): 2.93(6 \mathrm{H}, \mathrm{s}), 3.03(6 \mathrm{H}, \mathrm{s}), 6.05(1 \mathrm{H}, \mathrm{t}, J=2.0 \mathrm{~Hz}), 6.39(2 \mathrm{H}, \mathrm{dd}, J=2.0,8.0 \mathrm{~Hz}), 6.60$ $(2 \mathrm{H}, \mathrm{dd}, J=1.7,7.8 \mathrm{~Hz}), 6.70(1 \mathrm{H}, \mathrm{t}, J=8.0 \mathrm{~Hz}), 6.75(2 \mathrm{H}, \mathrm{dt}, J=1.7,7.8 \mathrm{~Hz}), 6.84(2 \mathrm{H}, \mathrm{dt}, J=1.5$, $7.6 \mathrm{~Hz}), 7.22(2 \mathrm{H}, \mathrm{dd}, J=1.5,7.9 \mathrm{~Hz}) .{ }^{13} \mathrm{C} \mathrm{NMR}\left(75 \mathrm{MHz}, \mathrm{CDCl}_{3}\right) \delta(\mathrm{ppm}): 38.3,39.5,122.4,122.6$ 122.7, 127.3, 127.5, 128.9, 129.7, 132.9, 143.4, 145.3, 160.0. MS (APCI+) : $561\left(\mathrm{MH}^{+}, 100\right)$. HRMS for $\mathrm{C}_{24} \mathrm{H}_{25} \mathrm{Br}_{2} \mathrm{~N}_{4} \mathrm{O}_{2}\left(\mathrm{MH}^{+}\right)$: calcd : 559.0339; found : 559.0333. Elem. Anal. for $\mathrm{C}_{24} \mathrm{H}_{24} \mathrm{Br}_{2} \mathrm{~N}_{4} \mathrm{O}_{2}$ : calcd: C, 51.45\%; H, 4.32\%; Br, 28.52\%; N, 10.00\%; found : C, 51.55\%; H, 4.28\%; Br, 28.44\%; N, 9.84\%.

$N, N$, $N$ ', $N$ ','-Tetramethyl-1,3-di-(2,6-diethylphenylureyl)benzene 17e. -In a similar way, 1,3phenylene diisocyanate $(500 \mathrm{mg}, 3.12 \mathrm{mmol}, 1 \mathrm{eq})$ and 2,6-diethylaniline (1.08 $\mathrm{mL}, 6.55 \mathrm{mmol}, 2.1 \mathrm{eq})$ gave, after methylation in THF (30 mL) with $\mathrm{NaH} 60 \%$ in mineral oil (624 mg, $15.6 \mathrm{mmol}, 5$ eq.) and methyl iodide ( $970 \mu \mathrm{L}, 15.6 \mathrm{mmol}, 5$ eq.), crude material which was recrystallised in $\mathrm{Et}_{2} \mathrm{O} /$ petroleum ether to yield urea $17 \mathrm{e}(1.27 \mathrm{mg}, 79 \%)$ as a white powder. m.p. : $186^{\circ} \mathrm{C}$. IR $v_{\max } \mathrm{cm}^{-1}: 2998,2972$, 2872, 1644, 1598, 1453. ${ }^{1} \mathrm{H}$ NMR (300 MHz, $\left.\mathrm{CDCl}_{3}\right) \delta(\mathrm{ppm}): 1.05(12 \mathrm{H}, \mathrm{d}, J=7.5 \mathrm{~Hz}), 2.08(4 \mathrm{H}, \mathrm{m})$, $2.28(4 \mathrm{H}, \mathrm{m}), 2.77(6 \mathrm{H}, \mathrm{s}), 3.01(6 \mathrm{H}, \mathrm{s}), 5.65(1 \mathrm{H}, \mathrm{s}), 6.38(2 \mathrm{H}, \mathrm{d}, J=7.2 \mathrm{~Hz}), 6.77(5 \mathrm{H}, \mathrm{m}), 6.94(2 \mathrm{H}$, $\mathrm{t}, J=7.5 \mathrm{~Hz}) .{ }^{13} \mathrm{C} \mathrm{NMR}\left(75 \mathrm{MHz}, \mathrm{CDCl}_{3}\right) \delta(\mathrm{ppm}): 14.1,23.3,38.4,40.7,123.7,123.8,125.7,127.2$, 128.5, 140.6, 141.2, 145.6, 161.6. MS (APCI+) : $515.4\left(\mathrm{MH}^{+}\right)$. HRMS for $\mathrm{C}_{32} \mathrm{H}_{43} \mathrm{~N}_{4} \mathrm{O}_{2}\left(\mathrm{MH}^{+}\right)$: calcd : 515.3381; found : 515.3379. Elem. Anal. for $\mathrm{C}_{32} \mathrm{H}_{42} \mathrm{~N}_{4} \mathrm{O}_{2}$ : calcd: C, 74.67\%; H, 8.22\%; N, 10.89\%; found : C, $74.18 \% ; \mathrm{H}, 8.20 \% ; \mathrm{N}, 10.82 \%$.

N,N',N',N",'Tetramethyl-1,3-di-(3-isopropylphenylureyl)benzene 17f. -In a similar way 1,3phenylene diisocyanate (500 mg, $3.12 \mathrm{mmol}, 1 \mathrm{eq}$ ) and 3-isopropylaniline (920 $\mu \mathrm{L}, 6.55 \mathrm{mmol}, 2.1 \mathrm{eq})$ gave, after methylation in THF (30 mL) with $\mathrm{NaH} \mathrm{60 \%} \mathrm{in} \mathrm{mineral} \mathrm{oil} \mathrm{(624} \mathrm{mg,} 15.6 \mathrm{mmol}, 5$ eq.) and 
methyl iodide $\left(970 \mu \mathrm{L}, 15.6 \mathrm{mmol}, 5\right.$ eq.) a crude product which was recrystallised from $\mathrm{Et}_{2} \mathrm{O} /$ petroleum ether to yield urea $\mathbf{1 7 f}(1.17 \mathrm{~g}, 77 \%)$ as a white powder. m.p. : $138^{\circ} \mathrm{C}$. IR $v_{\max } \mathrm{cm}^{-1}: 2960$, 2928, 1660, 1599, 1432, 1357, 1115. ${ }^{1} \mathrm{H} \mathrm{NMR}\left(500 \mathrm{MHz}, \mathrm{CDCl}_{3}\right) \delta(\mathrm{ppm}): 1.05(12 \mathrm{H}, \mathrm{d}, J=6.9 \mathrm{~Hz})$, $2.62(2 \mathrm{H}$, sept, $J=6.9 \mathrm{~Hz}), 2.95(6 \mathrm{H}, \mathrm{s}), 3.12(6 \mathrm{H}, \mathrm{s}), 5.99(1 \mathrm{H}, \mathrm{t}, J=2.0 \mathrm{~Hz}), 6.41(2 \mathrm{H}, \mathrm{dd}, J=2.0$, $8.0 \mathrm{~Hz}), 6.49(2 \mathrm{H}, \mathrm{s}), 6.53(2 \mathrm{H}, \mathrm{dd}, J=2.0,7.9 \mathrm{~Hz}), 6.71(1 \mathrm{H}, \mathrm{t}, J=8.0 \mathrm{~Hz}), 6.74(2 \mathrm{H}, \mathrm{d}, J=8.4 \mathrm{~Hz})$, $6.91(2 \mathrm{H}, \mathrm{t}, J=7.8 \mathrm{~Hz}) .{ }^{13} \mathrm{C}$ NMR $\left(125 \mathrm{MHz}, \mathrm{CDCl}_{3}\right) \delta(\mathrm{ppm}): 23.8,33.7,39.0,39.4,122.4,123.1$, 123.2, 123.3, 123.8, 128.1, 128.3, 145.3, 145.7, 149.3, 160.9. MS (ESI+) : $487\left(\mathrm{MH}^{+}, 100\right)$. HRMS for $\mathrm{C}_{30} \mathrm{H}_{39} \mathrm{~N}_{4} \mathrm{O}_{2}\left(\mathrm{MH}^{+}\right)$: calcd : 487.3068; found : 487.3074. Elem. Anal. for $\mathrm{C}_{30} \mathrm{H}_{38} \mathrm{~N}_{4} \mathrm{O}_{2}$ : calcd: C, 74.04\%; H, 7.87\%; N, 11.51\%; found : C, 73.85\%; H, 7.95\%; N, $11.44 \%$.

$N, N^{\prime}, N$, $N$ ','Tetramethyl-1,3-di-(3-ethylphenylureyl)benzene $\quad 17 \mathrm{~g} . \quad$ - In a similar way, 1,3phenylenediisocyanate (500 mg, $3.12 \mathrm{mmol}, 1 \mathrm{eq})$ and 3-ethylaniline (810 $\mu \mathrm{L}, 6.55 \mathrm{mmol}, 2.1 \mathrm{eq})$ gave, after methylation in THF (30 mL) with $\mathrm{NaH} 60 \%$ in mineral oil (624 mg, $15.6 \mathrm{mmol}, 5$ eq.) and methyl iodide $\left(970 \mu \mathrm{L}, 15.6 \mathrm{mmol}, 5\right.$ eq.) a crude product which was recrystallised from $\mathrm{Et}_{2} \mathrm{O} /$ petroleum ether to provide urea $17 \mathrm{~g}(1.08 \mathrm{~g}, 76 \%)$ as a white powder. m.p. : $137^{\circ} \mathrm{C}$. IR $v_{\max } \mathrm{cm}^{-1}: 2963,2931,2874$, 1659, 1599, 1352, 1116. ${ }^{1} \mathrm{H}$ NMR $\left(500 \mathrm{MHz} \mathrm{CDCl}_{3}\right) \delta(\mathrm{ppm}): 1.04(6 \mathrm{H}, \mathrm{d}, J=7.5 \mathrm{~Hz}), 2.38(4 \mathrm{H}, \mathrm{q}, J$ $=7.5), 2.95(3 \mathrm{H}, \mathrm{s}), 3.11(3 \mathrm{H}, \mathrm{s}), 6.01(1 \mathrm{H}, \mathrm{t}, J=2.1 \mathrm{~Hz}), 6.41(2 \mathrm{H}, \mathrm{dd}, J=2.0,7.0 \mathrm{~Hz}), 6.46(2 \mathrm{H}, \mathrm{s})$, $6.54(2 \mathrm{H}, \mathrm{dd}, J=1.0,8.0 \mathrm{~Hz}), 6.71(2 \mathrm{H}, \mathrm{d}, J=8.0 \mathrm{~Hz}), 6.73(1 \mathrm{H}, \mathrm{t}, J=8.0 \mathrm{~Hz}), 6.90(2 \mathrm{H}, \mathrm{t}, J=7.8$ Hz). ${ }^{13} \mathrm{C}$ NMR $\left(125 \mathrm{MHz}, \mathrm{CDCl}_{3}\right) \delta(\mathrm{ppm}): 15.3,28.4,39.0,39.3,122.3,123.0,124.5,125.3,128.2$, 128.3, 144.6, 145.3, 145.7, 160.8. MS (APCI+) : $459\left(\mathrm{MH}^{+}, 100\right)$. HRMS for $\mathrm{C}_{28} \mathrm{H}_{35} \mathrm{~N}_{4} \mathrm{O}_{2}\left(\mathrm{MH}^{+}\right)$: calcd : 459.2755; found : 459.2754. Elem. Anal. for $\mathrm{C}_{28} \mathrm{H}_{34} \mathrm{~N}_{4} \mathrm{O}_{2}$ : calcd: C, 73.33\%; H, 7.47\%; N, 12.22\%; found : C, 73.80\%; H, 7.56\%; N, $12.25 \%$. 


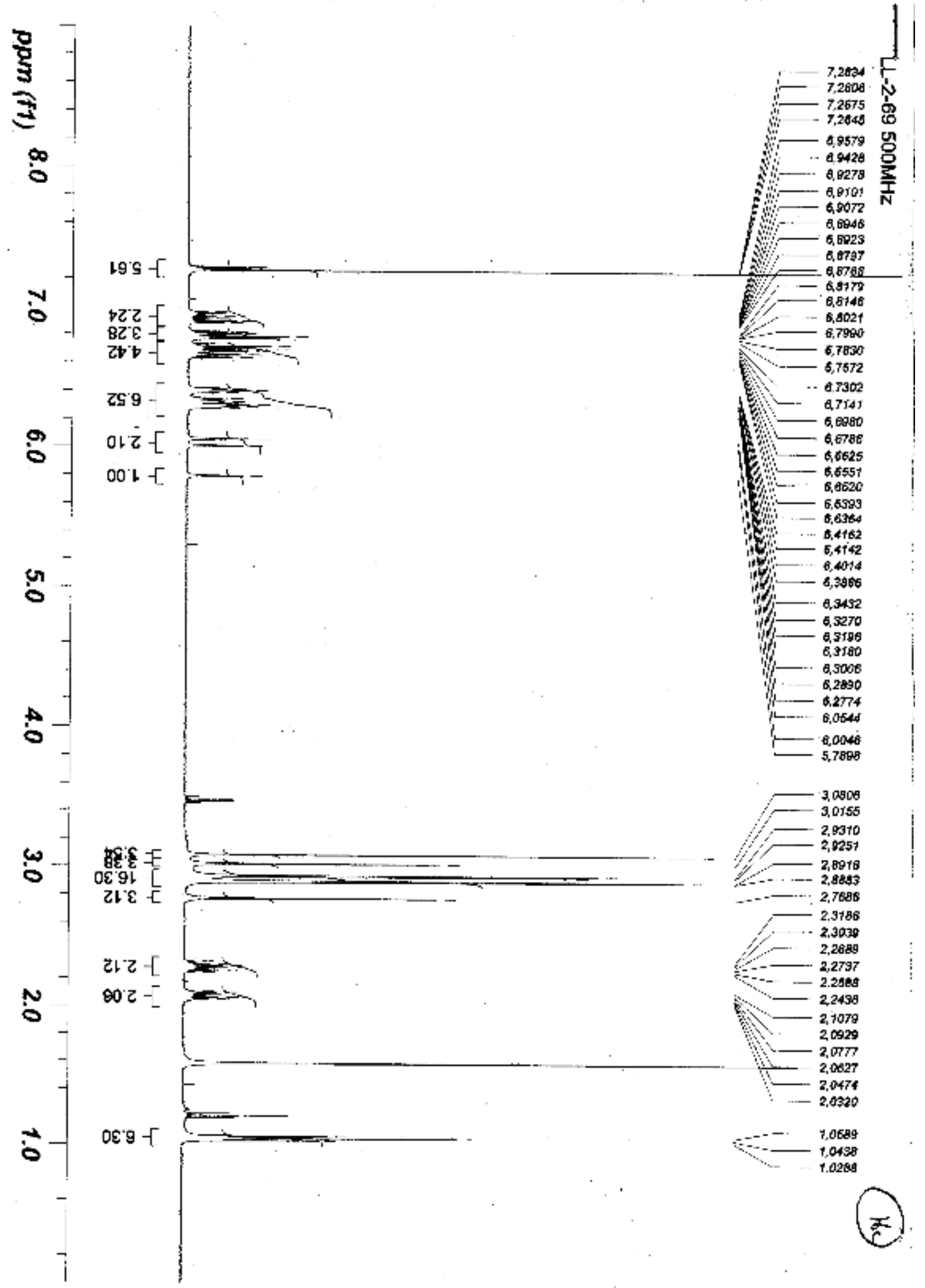




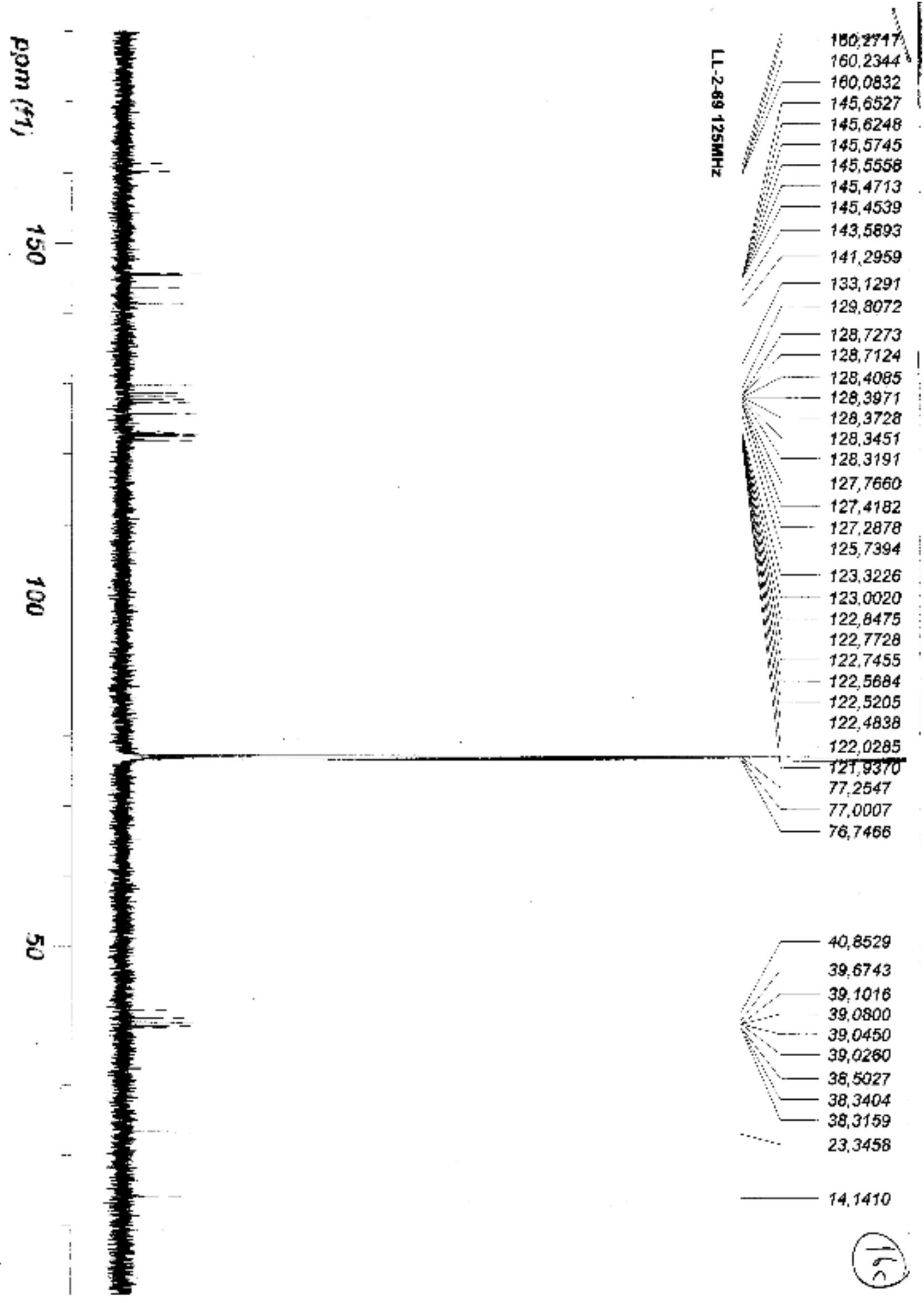




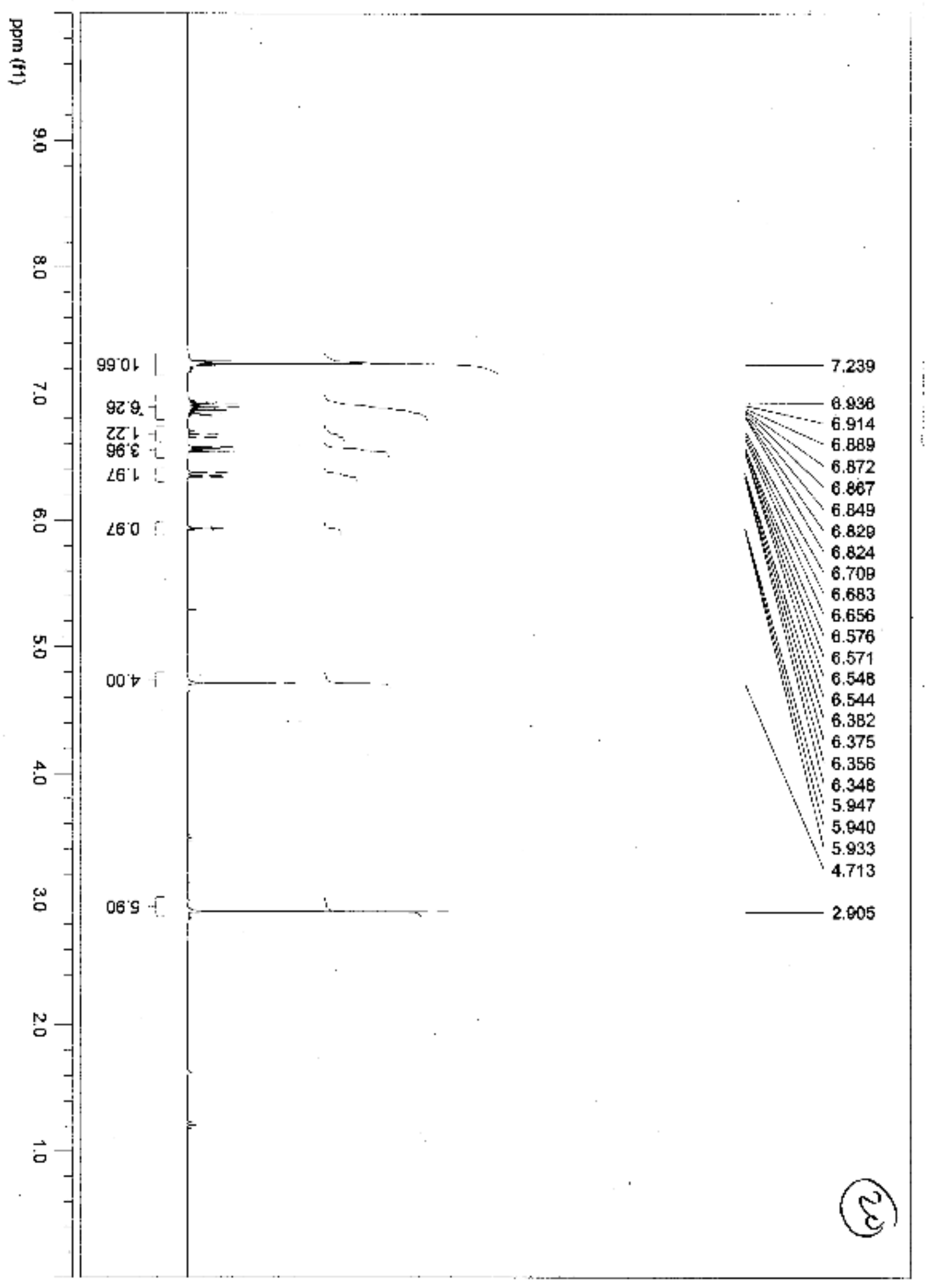




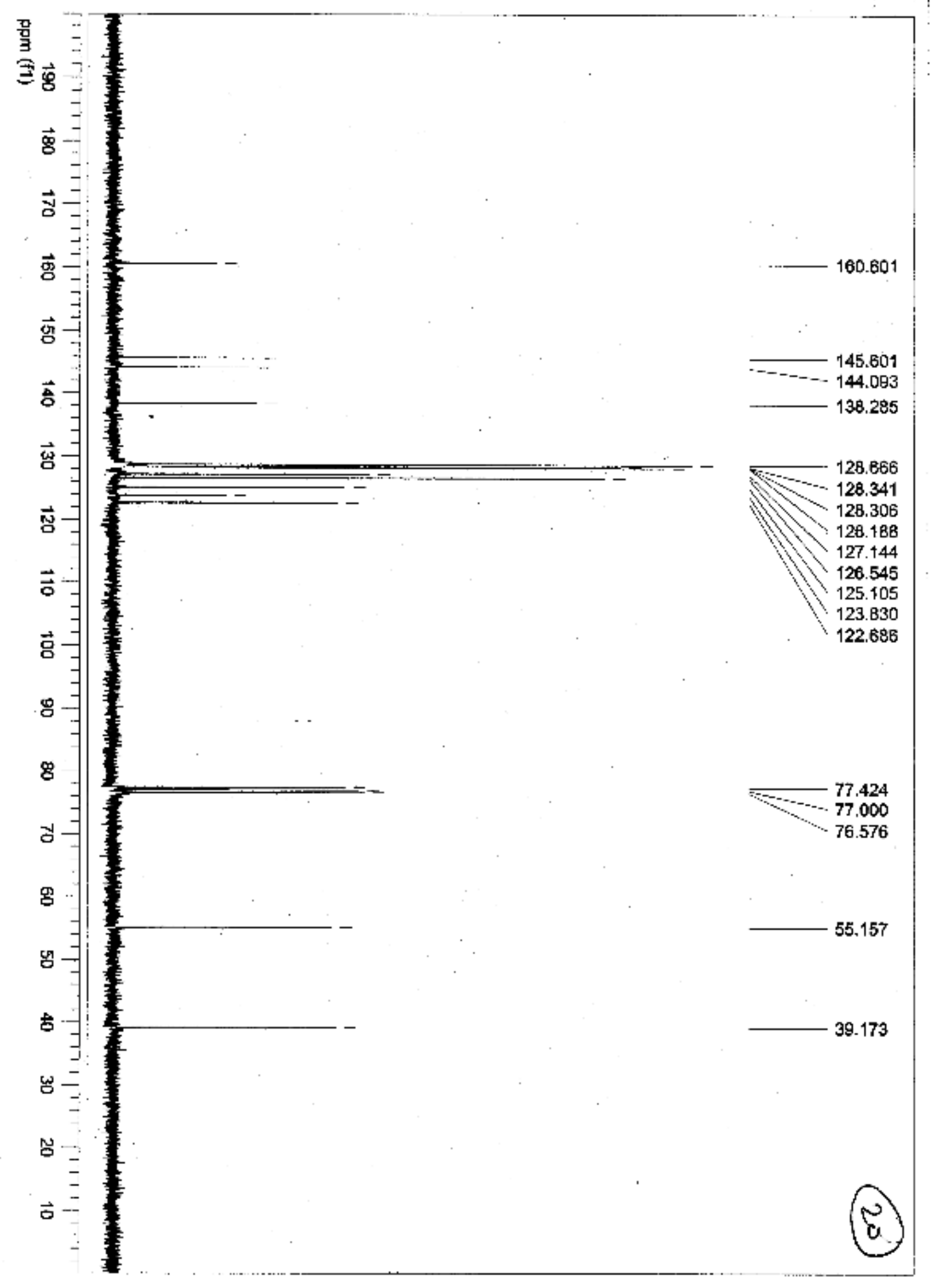

